\title{
La pequeña y mediana industria metalmecánica ante el proceso de apertura petrolera
}

\author{
Caterina Clemenza* \\ Angel Urbina** \\ Rosmary Rodríguez*** \\ Alba Roncancio*** \\ Liliana Roncancio***
}

\section{Resumen}

En el mercado venezolano, como consecuencia de la expansión petrolera se están generando nuevas oportunidades de participación de las empresas metalmecánicas. En los últimos años se ha evidenciado como la pequeña y mediana industria metalmecánica (PyMIMM) ha dirigido sus esfuerzos a suplir las necesidades de la industria petrolera nacionales. Se realiza esta investigación con el objetivo de determinar si la PMIMM posee las condiciones necesarias que le permitan adaptarse a las nuevas exigencias. El tipo de investigación es descriptiva. Se realizó un censo am 30 pequeños y medianos industriales metalmecánicos, localizados en los Municipios Maracaibo y San Francisco del Estado

\section{Recibido: 05-11-97 . Aceptado: 20-12-97}

* Economista. Magister en Gerencia de Empresas. Investigadora - Profesora Agregado a Dedicación Exclusiva. Universidad del Zulia. Facultad de Ciencias Económicas y Sociales. Instituto de Investigaciones. Departamento de Estudios Regionales y Urbanos. Maracaibo - Estado Zulia Venezuela. Teléfonos Fax:00 - 58-61 596513,00 - 58 - 61975738, Hab: 00 - 58 - 61533156 E-Mail: cclemenz Qeuropa.ica.luz.ve. Favor enviar correspondencia a la siguiente dirección: Res. La Puerta de Ciudadela Faria. Edif. La Mesa. Apto. 5 - 3. Maracaibo-Estado Zulia.

* Economista. Profesor - Investigador. Universidad del Zulia. Facultad de Ciencias Económicas y Sociales. Instituto de Investigaciones. Departamento de Estudios Regionales y Urbanos. Maracaibo - Estado Zulia.

** Tesistas de pre-grado. Colaboradoras del proyecto "Impacto de la Apertura Petrolera", financiado por el CONDES, del cual forma parte esta investigación. 
Zulia. Se aplicó una entrevista semiestructurada; además se recurrió a la revisión y análisis de documentos y estadística nacional y regional relacionada con el sector. Como conclusión, debe lograrse que las PyMIMM desarrollen una gerencia profesional ágil, capaz de generar cambios y transformaciones, además de una mano de obra calificada y un parque tecnológico que responda a las exigencias de cambio que se están operando en nuestro pais.

Palabras clave: Apertura Petrolera, Sector Industrial, Pequeña y Mediana Industria Metalmecánica, Gestión Tecnológica, Organización Gerencial .

\section{Small and Medium Sized Metalurgical Industries and the Economic Opening of the Oil Industry}

\section{Abstract}

The venezuelan market, as a consequence of the expansion of the oil industry, is offering new possibilities of participation for industries in the metalurgical sector. In the last few years it is easily observable that the small and medium sized metalurgical companies (PyMIMM) have directed their efforts to fulfilling the needs of the national oil industry. This research is being done in order to determine if the PyMIMM has the necessary resources which permit it to adapt to the new requirements which are demanded by this process. It is a descriptive study. 30 companies belonging to the PyMIMM located in the Municipalities of Maracaibo and San Francisco was taken; A semi-structured interview survey was used to collect the information, as well as analysis of documents and national and regional statistics related to the topic. The study concludes that, an agile, professional management, capable of change and transformation is required, as well as qualified labor and a technological park which can respond to the needs and changes which are operating in the country.

Key words: Economic liberalization of the Oil industry, Small and Medium sized Metalurgical Industry, Technological management, Organizational management.

\section{Introducción}

El proceso de industrialización venezolana alcanzó durante las primeras décadas de la segunda mitad del presente siglo, una fase de crecimiento, consolidación y relativa estabilización, por la política industrial de sustitución de importaciones (años 1950-1960) que dejó al país un parque industrial importante, el cual hasta finales de los años 80 , aún presentaba cierto adelanto tecnológico con respecto al resto de América Latina. Además, con la intervención estatal se fijó en el proceso industrial un cierto énfasis sectorial, que se inicia con el establecimiento de industrias básicas en el área de la petroquímica, plásticos, caucho y metalmecánica, ésta última impulsada fundamentalmente por el liderazgo de las ensam- 
bladoras automotrices que se establecieron en Venezuela.

En el caso concreto de la pequeña y mediana industria, éstas han tenido un papel importante en el desarrollo económico del sector industrial en Venezuela, al producir ciertos bienes que las grandes industrias no están dispuestas a producir por la baja demanda en el mercado. La producción en la pequeña y mediana industria requiere el uso intensivo de mano de obra, razón por la cual el desarrollo de este segmento determina en buena medida los niveles de empleo y bienestar en el país, no siendo este aspecto el único en el cual se manifiesta la contribución de dicho sector.

Dentro del contexto empresarial de la pequeña y mediana industria en general, existen insuficiencias gerenciales, organizativas y técnicas de las empresas, que impiden hacer uso óptimo de las capacidades humanas y de equipamiento disponibles para mejorar la productividad e incentivar el desarrollo de cambios tecnológicos y organizativos de las empresas. El sector de la pequeña y mediana industria metalmecánica (PyMIMM), no ha escapado a esta problemática general que afecta a todas las pequeñas y medianas industrias del país. Lo concerniente al mercado, ha sido la constante escasez de demanda frente a las potencialidades de la oferta, la generación persistente de excedentes de la capacidad instalada ante una progresiva contracción en el nivel de empleo; y los problemas del lado de la oferta, como son los crecientes costos energéticos y laborales, el valor de la materia prima y de los gastos financieros; las altas tasas de interés actualmente vigentes en el mercado financiero bancario, hacen imposible que la PyMIMM, enfrente los precios de la materia prima, debido a los elevados costos que representa la obtención del dinero vía crediticia.

Uno de los roles más significativos de la PyMIMM, es cumplir como proveedor excelente, estable y confiable de partes y componentes para las grandes empresas, teniendo en la mayoría de los casos relaciones estables y de largo plazo de subcontratación. La pequeña y mediana industria metalmecánica conforma un gran apoyo para todas las grandes empresas existentes tanto en la región como en el país, especialmente como suplidora de las empresas petroleras.

En Venezuela, se están abriendo las puertas al capital privado extranjero y nacional a través del Proyecto de Apertura Petrolera, este proceso atraerá al país un caudal significativo de inversión y tecnología foránea de utilidad para el desarrollo de empresas nacionales.

La apertura petrolera puede convertirse en un hecho histórico importante, que marque de manera definitiva una nueva relación del petróleo con el país y que provea a éste de una necesaria imagen internacional positiva para las inversiones privadas, ya sean nacionales o extranjeras.

Con este proceso se estimulará el desarrollo de empresas nacionales, pues está establecido que los inversionistas deberán adquirir bienes y servicios producidos en el pais, siempre y cuando éstos sean competitivos en calidad, precio y tiempo de entrega.

Dando respuesta a estos lineamientos, Petróleos de Venezuela ha fijado políticas para la participación del sector privado nacional en los diferentes seg- 
mentos del negocio. De tal manera que con la Tercera Ronda de Convenios Operativos, en donde se han reactivadoe incorporado campos inactivos a la producción de crudos venezolanos, el sector privado nacional tiene la oportunidad de participar directamente de los beneficios, por cualquiera de las siguientes modalidades: participación como empresas licitantes, participación financiera a través de la Sociedad de Fomento de Inversiones Petroleras (SOFIP) y como empresas suplidoras de bienes y servicios.

En este último aspecto, es donde la PyMIMM tiene una excelente oportunidad, ya que la industria petrolera tiene una integración orgánica con el aparato productivo nacional, mucho más intensa que la mayoría del sector manufacturero. Esto se evidencia al observar las cifras que indican que para 1996 , un $60 \%$ de los equipos y materiales y cerca de un $90 \%$ de los senvicios requeridos por la industria petrolera se compran o se contratan en el país.

Con referencia a la participación del sector petrolero en la industria nacional, en el informe anual de PDVSA se plantea que para 1996, algo más de un millardo fue transferido al sector privado (PDVSA, 1997), a causa de las compras realizadas por PDVSA de productos nacionales y productos importados por firmas venezolanas, que luego fueron vendidos a la industria petrolera. En el mismo informe se señala, que el sector petrolero privado ha desarrollado en los últimos años el sector productivo venezolano a través de la adquisición de bienes y servicios.

Para 1996 se destinó un total de 941.288 millones de bolívares por con- cepto de adquisición de materiales y equipos. De esta suma 544.973 millones correspondieron a la compra de productos nacionales e importados por firmas venezolanas, y 395.316 millones se destinaron a productos que fueron importados directamente por la industria. (PDVSA, 1997).

Lo antes expuesto lleva a reflexionar sobre la necesidad de desarrollar el sector industrial nacional para que le sirva de apoyo a Petróleos de Venezuela, así como al sector petrolero privado.

En este orden de ideas son enormes las posibilidades que ofrecen los sectores metalmecánicos, de bienes de capital, material de transporte, construcción, servicios petroleros y de ingeniería, entre otros, capaces de responder a los impulsos de una inversión y crecimiento como el que se deriva del programa petrolero. Por ejemplo, en el área de la producción nacional de bienes de capital para la industria petrolera, se mencionan los siguientes: tanques de almacenamientos de todos los tamaños, recipientes de presión, tubería de pequeño y gran diámetro, válvulas de todo tipo y tamaño, bridas, conexiones, mechas de perforación, bombas, compresores, tableros y transformadores, entre otros.

El sector metalmecánico que agrupa un poco más de 1.000 compañías, actualmente trabaja con el $40 \%$ de su capacidad instalada de manufactura. PDVSA y sus filiales requieren suministro de equipos y bienes de capital. La intensión de las petroleras es adquirir el máximo de esos insumos disponibles en el pais, 10 cual implica que las empresas metalmecánicas nacionales deben operar al 
$100 \%$ de su capacidad instalada de la producción (Paredes, 1997:E1).

Esta oportunidad debe ser aprovechada por el sector industrial metalmecánico; para ello se debe desarrollar técnica y gerencialmente a fin de hacer frente a todas las demandas del sector petrolero de manera eficiente. Por esto se requiere una gerencia profesional, ágil y capaz de generar cambios y transformaciones, además de una mano de obra calificada y un parque tecnológico que responda a las exigencias de cambio que se están operando en el país.

\section{Pequeña y mediana industria metalmecánica. Algunas consideraciones}

A continuación se presentan algunos aspectos referidos a la PyMIMM, con el fin de tener una idea global de éstas.

La industria metaimecánica abarca todos aquellos sectores productivos que se dedican a la transformación de metales. Se incluyen dentro de éste subconjunto manufacturero tanto las plantas de fundición y forja como los talleres de estampado, corte y soldadura, tratamiento térmico, de metale,s diversos; también el sector agrupa los establecimientos de armado $y$ ensamble final de maquinaria eléctrica y no eléctrica, de vehículos y materiales de transporte y de equipos varios de indole científica. (Katz, 1983: 90).

En esta industria participan dos tipos de industrias: fabricantes terminales de maquinarias y equipos y fabricantes de partes y piezas. Los primeros se dedican al ensamble de productos y a la elaboración de los componentes más criticos o especiales y los segundos son proveedores de componentes especifi$\cos$. De alli que desde el punto de vista industria y tecnológico estos dos tipos de fabricantes conforman un conjunto industrial. (Cepal, 1989: 5).

La tecnología de estas ramas productivas posee una gama de rasgos peculiares que sin duda afectan la conducta tecnológica de la empresa, vale la pena mencionar: a) El gran número y la enorme diversidad de subprocesos necesarios para fabricar un producto metalmecánico dado, b) La amplitud y complejidad del árbol de componentes que liga piezas, submontajes y productos finales, c) La universalidad o carácter de uso múltiple, que posee una parte mas o menos importante del equipamiento utilizado por el sector. d) El alto grado de sustituibilidad entre subprocesos y técnicas productivas.

La producción metalmecánica abarca una gama sumamente heterogénea de productos a los que se llega a través de una extensa nómina de subprocesos. La industria metalmecánica se caracteriza también por la complejidad del árbol de componentes que liga a piezas, submontajes y productos; ocurre que todo producto final complejo, por ejemplo, un automóvil, es la suma de una serie de subconjuntos, cada uno de los cuales requiere a su vez un montaje propio efectuado con anterioridad. Las diferencias entre las industrias de proceso y las ramas metalmecánicas, por supuesto, no acaban con el mayor número de subprocesos que normalmente integran la tecnología de estás últimas, ni con la mayor complejidad y amplitud del árbol de componentes y las opciones de descentrali- 
Industria metalmecánica y apertura petrolera

Caterina Clemenza y col.

zación y especialización que éste trae aparejadas.

Las plantas metalmecánicas de América Latina están lejos de constituir una réplica de los establecimientos fabriles productores de bienes semejantes en paises industriales maduros. Para explorar las diferencias más notorias tanto en el plano teórico como en el de la formulación de instrumentos de política pública; será necesario recalcar aspectos tales como: a) El tamaño de la planta fabril. b) La nacionalidad y su modelo organizativo. c) La edad y el grado de madurez tecnológico de sus departamentos técnicos. d) Los rasgos morfológicos del mercado que abastece (Monopolio o competitivo). e) Los mercados de factores donde se provee de insumos. f) El marco legal e institucional en que opera, etc.

Las diferencias de madurez dentro de la región latinoamericana son sumamente marcadas. Algunos paises como Argentina y Brasil comenzaron a desarrollar su industria metalmecánica relativamente temprano a comienzos de este siglo. Muchas de ellas han emergido de talleres de mantenimiento y reparación operados por distribuidores e importadores de productos extranjeros. Los parques automotores y de bienes de consumo duradero, de dichos países, eran en ese momento, grandes, aún si se los compara con los de naciones del mundo desarrollado (Katz, $1983: 100$ ).

Durante los años 30 se radican en dichos paises diversas subsidiarias de grupos metalmecànicos de naciones desarrolladas. Ellas no eran, en aquel momento unidades de producción propiamente dichas, sino casas distribuidoras y de representación comercial. Resulta evidente el hecho de que varias de estas firmas montan departamentos de asistencia técnica a clientes, así como también de reparación y mantenimiento del parque local respectivo.

En otros países de la región el surgimiento de actividades metalmecánicas es de data más reciente. Puede situarse en la década de los años 50 en México, Colombia y Chile y al término de los sesenta, o al comienzo de la década siguiente, en Venezuela o Perú.

Dichas diferencias de edad en las respectivas industrias metalmecánicas de la región se traducen en diferencias madurativas de importancia; ello implica que la industria metalmecánica brasileña o argentina se encuentra actualmente en condiciones de operar a base de un paquete de información técnica más sofisticado y complejo que por ejemplo, la rama metalmecánica de Venezuela, Perú, Argentina y Brasil; $y$ en menor medida México, Colombia, muestran la existencia de diversas ramas metalmecánicas en las que es común observar la presencia de plantas de proceso continuo, donde el trabajo está organizado en línea como corresponde a programas de producción masiva destinada a grandes mercados. Lo importante es que sólo algunos países de la región, típicamente Brasil, Argentina, México y Colombia revelan haber desarrollado gradualmente, en distintas plantas de su industria metalmecánica, capacidad interna de ingeniería.

Al tratar de examinar la tecnología metalmecánica de la región Latinoamericana, se observa un complejo mosaico de diferencias técnicas entre establecimientos fabriles. Dichas diferencias son importantes no sólo en el interior de cada 
país y entre paises de la región, sino también entre establecimientos de la región y plantas industriales del mundo desarrollado. (Katz, 1983: 101).

Venezuela al igual que Perú y en comparación con otros países latinoamericanos como Argentina, Brasil, México y Colombia, tiene una historia empresarial breve en el sector metalmecánico que en algunos casos, data de inicios de los años 70 (Katz, 1983: 87-88).

Ampliando esta información Bitary Troncoso ilustran la evolución que ha tenido este sector en la economia nacional $y$ al respecto indican:

La industria fue creándose poco a poco.

Inicialmente se instalaron talieres para reparación y mantenimiento y luego se establecieron unidades fabricantes de estructuras metálicas simples... se extendió mas tarde a recipientes, calderas, algunos motores, generadores, bombas y otros artículos de usos generalizados, pero... sin un plan previo ni una politica deliberada, el mayor impulso al sector surgió en la década del 70 , de la industria automotriz y de los electrodomésticos. (Bitar y Troncoso, 1983:168).

La política de sustitución de importaciones, impactó positivamente el desarrollo del sector metalmecánico productor de bienes de capital. En buena medida se asocian con esta política los esfuerzos destinados al establecimiento de industrias que suplieran las necesidades de materiales, herramientas, maquinarias y equipos para el parque industrial. No obstante puede indicarse que en general la evolución del sector ha sido lenta al igual que el resto del sector manufacturero (Ferrer, 1990: 25).

Dos hechos han producido el desarrollo de éste sector económico en la región: la influencia de la actividad petrolera y la política de sustitución de importaciones. Algunos datos obtenidos de la documentación revisada indican que: " ... el Zulia cuenta con una industria perfeccionada, con unos estándares de calidad muy elevados, por cuanto la misma nació y se hizo fuerte a través del suministro de bienes, equipos, partes y piezas destinadas al sector petrolero" (Ferrer, 1990:30).

\section{Características generales de la pequeña y mediana industria metalmecánica (PYMIMM)}

A continuación se presentan los resultados de las entrevistas realizadas en 1997 a los pequeños y medianos industriales ubicados en el Municipio Maracaibo y San Francisco inscritos en el Comité Empresarial de la zona Industrial de Maracaibo (CEZIMAR) y la Cámara de Industriales del Zulia, durante 1996, con el propósito de analizar la gestión tecnológica, y la capacidad profesional y técnica de la mano de obra empleada en la pequeña y mediana industria metalmecánica que ellos dirigen, a fin de determinar si están preparados técnica y gerencialmente para enfrentar el reto que significa competir en precios, calidad y tiempo de entrega bajo la nueva realidad económica que, con el proceso de Apertura Petrolera, está surgiendo en el país y por ende en la Región Zuliana. 
Para el conjunto de PyMIMM seleccionada para el estudio, se determinó que éstas desarrollan diferentes actividades dentro de las subramas de las industrias metalmecánicas. Dentro de las mismas se concentran las empresas que fabrican maquinarias y equipos para la industria, es decir, bienes de capital, representando un $53 \%$ del total de las industrias objeto de estudio. Tal resultado, indica que estas empresas son las que mayor apoyo le brindan a la industria petrolera nacional y a las empresas conexas a ellas, así como también otras empresas del sector.

Además, se puede observar que el $30 \%$ de la PyMIMM, se agrupa en la subrama de productos metálicos, desarrollando especificamente actividades re- lacionadas con productos estructurales; el resto $(17 \%)$, integran la subrama de materiales y equipos eléctricos, destacándose la fabricación de tableros eléctricos, y equipos de control de calidad. (Gráfico No. 1)

Para desarrollar sus procesos productivos, el $55 \%$ de las PyMIMM estudiadas utilizan maquinarias importadas y el $53 \%$ insumos importados demostrándose con estas cifras, que un poco mas de la mitad de las industrias recurren al mercado internacional para adquirir la tecnología que necesitan y para abastecerse de materia prima para la elaboración de sus productos.

Sin embargo, el número de PyMIMM que utilizan maquinarias e insu-

\section{Gráfico No. 1}

Municipios Maracaibo y San Francisco

Pequeña y Mediana Industria Metaimecanica

Tipo de Subrama a la cual pertenece

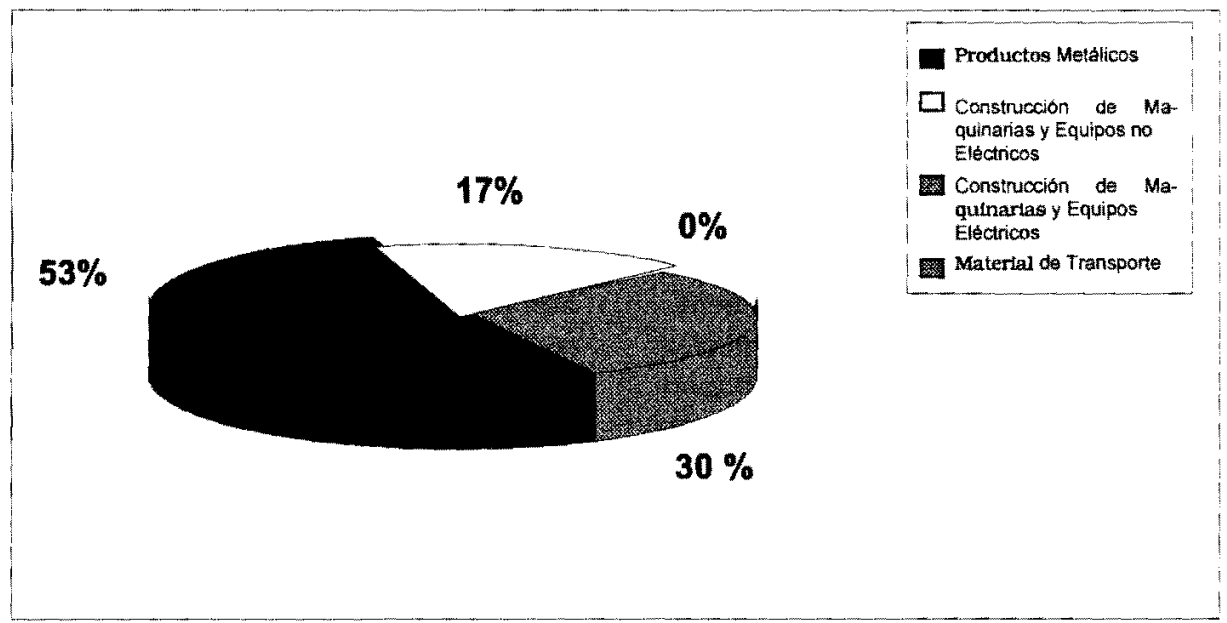

Fuente: Encuesta realizada en los Municipios Maracaibo y San Francisco, Mayo 1997. Cálculos Propios 
mos nacionales es significativo $(45 \%$ y $47 \%$ ), lo cual lleva a pensar que existe un parque industrial nacional que le sirve de apoyo a estas industrias y que puede competir con las empresas internacionales.

Según las encuestas realizadas, el mayor número de trabajadores ocupados, en las industrias metalmecánicas, está concentrado en la mediana industria, representando ésto un $67 \%$, lo cual indica que de 30 industrias, 20 de éstas poseen de 21 a 100 trabajadores. El restante $33 \%$ está concentrado en la pequeña industria. Se concluye que el mayor estrato se encuentra en la mediana empresa, aunque más cerca del límite superior.

Del total de las empresas encuestadas, se observa que la participación de los profesionales y técnicos es realmente significativa, los cuales en total suman un $44 \%$. Es decir, casi la mitad de los trabajadores de éstas industrias son de alta capacitación (por lo menos con tres años de educación superior).

Es importante destacar la presencia de los obreros calificados con un $31 \%$ del total de los trabajadores y con una participación al doble que la de los obreros no calificados (14\%).

Esta es, definitivamente, una de las razones clave: se está avanzando hacia una efectiva profesionalización de la pequeña y mediana industria metalmecánica. Por otro lado se puede considerar baja la participación del personal administrativo con un $11 \%$ del total; se constata así que no sólo hay una participación importante del personal altamente capacitado, sino también hay poca presencia del personal no directamente productivo.
En este sentido, la calificación de la mano de obra es uno de los factores de mayor importancia para el incremento de la productividad, de allí se hace necesaria la capacitación y fortalecimiento permanente tanto de los empresarios como de los operarios en gestión administrativa, financiera, producción, tecnología, comercialización, mercadeo y ventas.

Al respecto, cabe mencionar que para enfrenar el reto que supone para el Zulia el nuevo plan petrolero y aprovechar las oportunidades que se están presentando para el desarrolio de la PyMIMM, se debe definir como política, el fortalecimiento del empresario nacional vinculado a todas las fases de la economía petrolera, así como también dirigir los esfuerzos hacia la capacitación del personal técnico y administrativo.

\section{La PyMIMM y su relación con la industria petrolera}

La pequeña y mediana industria metalmecánica, proveedora de bienes y servicios a las industrias petroleras, representa un $94 \%$, lo cual significa que de las 30 empresas objeto de estudio 28 afirmaron ser proveedoras de éstas.

Por otra parte, se observa que el mayor porcentaje de las pequeñas y medianas industrias metalmecánicas proveedoras de las filiales petroleras, se concentran en Lagoven con un $30 \%$, Maraven con un $27 \%$ y Pequiven con $25 \%$, lo cual arroja un total de $82 \%$ y el $18 \%$ restante suple a las filiales Corpoven, Deltaven y Carbones del Guasare.

En el gráfico No. 2 se indica que el $65 \%$ de las industrias analizadas se concentran en la fabricación de maquinarias 
Gárfico No. 2

Municipios Maracaibo y San Francisco

Pequeña y Mediana Industria Metalmecanica

Sugún Tipo de Bienes o Servicios Ofrecidos

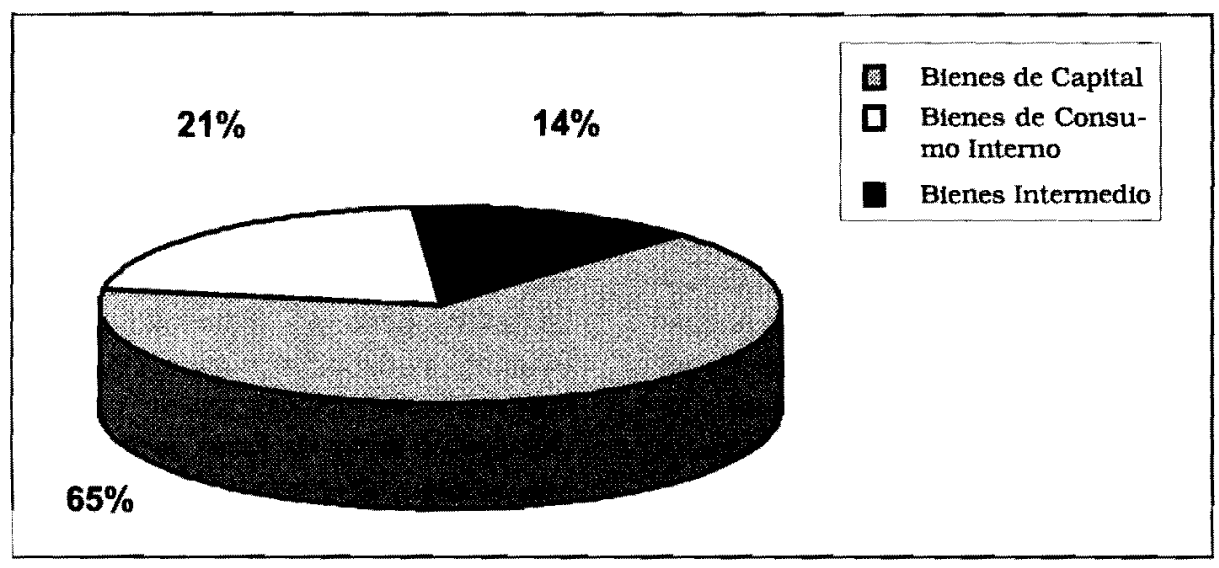

Fuente: Encuesta realizada en los Municipios Maracalbo y San Francisco, Mayo 1997. Cálculos Propios.

y equipos para la industria petrolera $y$ otros sectores económicos, es decir, bienes de capital, los cuales son esenciales para el desarrollo nacional, en particular para el desarrollo de la propia PyMIMM. Por lo tanto las maquinarias y equipos producidos por la metalmecánica juegan un papel importante en la generación de tecnología interna, permitiendo la creación de nuevos productos o mejorando los ya existentes.

Los resultados arrojados permiten comentar que la apertura petrolera representa un reto para que las industrias proveedoras privadas nacionales, se tornen mas competitivas. La política de contratación señala que los bienes y servicios serán adquiridos y contratados mediante licitaciones públicas o privadas..., en caso de existir ofertas nacionales comparables con las ofertas internacionales en términos de costos, calidad y tiempo de entrega, la empresa operadora contratará la oferta nacional (PEÑALOZA, 1996:36).

Los proveedores nacionales de bienes y servicios a la industria petrolera deben superar grandes dificultades para integrarse al proceso de apertura. Especificamente, la PYMIMM, ha venido padeciendo en los últimos años una recesión que ha limitado su desarrollo y su capacidad competitiva. Además éstas industrias se apoyan en un sector financiero local que las asiste comercialmente, pero que tiene una escasa capacidad para ofrecer financiamiento de mediano y largo plazo. 
De las 28 empresas que manifestaron tener relaciones con la industria petrolera el $89 \%$ afirmaron conocer los aspectos generales referidos al proceso de apertura petrolera, mientras que el $4 \%$ señaló que no estaban en conocimiento del mismo.

Por otra parte, los entrevistados indicaron que se estaban preparando para poder enfrentar todo to que significa el proceso de apertura petrolera. Para ello están planificando las estrategias necesarias, a fin de lograr competir con las empresas nacionales y extranjeras.

Es de esperarse, que ante el incremento previsto en la actividad de la industria petrolera, el mercado ofrezca grandes oportunidades a las PyMIMM. Estas deben diseñar estrategias a mediano y largo plazo, orientadas a generar la competitividad requerida para lograr una participación creciente en el mercado. Las estrategias que van a requerir mayor énfasis son las relacionadas con la actualización tecnológica, el aumento en la capacidad de ejecución y el acceso a las fuentes de financiamiento. Por otra parte, deben tener el propósito de asumir los riesgos, efectuar inversiones y mejorar técnica, comercial y gerencialmente para ofrecer a tiempo los bienes que producen y que éstos sean de calidad y con precios competitivos internacionalmente.

Los industriales entrevistados manifestaron en un $60 \%$ mantener relaciones con empresas de otras ramas, el $35 \%$ con industrias de la misma rama y el $5 \%$ con las subramas.

Por otra parte, estas PyMIMM se interrelacionan con las otras industrias en un $31 \%$ a través de convenios forma- les e informales, $29 \%$ por medio de venta directas e indirectas, el $25 \%$ a través de subcontratación, el resto manifestó, entre otros, realizar acuerdos para repartirse el mercado.

En este sentido, se puede afirmar que las PyMIMM que tienen relación directa con la industria petrolera ocasionan un impacto de arrastre significativo sobre el resto de las pequeñas, medianas y grandes industrias que están directa o indirectamente conectadas a ellas. Se produce un efecto encadenante entre el sector petrolero, las PyMIMM y el resto de las industrias.

La compra de bienes, equipos y materiales, así como la contratación de servicios de ingeniería producen un efecto horizontal que permitirá un desarrollo dinámico de este sector conexo a la industria petrolera.

Para que puedan lograr el objeto planteado, estas empresas deben, entre otras cosas conocer el mercado nacional e internacional, en el cual están inmersas Con respecto a esto, el $29 \%$ de los empresarios respondieron que conocian a sus clientes actuales y potenciales el $25 \%$ indicaron saber quienes son sus competidores y cuál es la tendencia tecnológica mundial y nacional, mientras que el $20 \%$ afirmó conocer las regulaciones fundamentales que rigen su negocio.

\section{Gestion tecnológica y gerencial}

El $96 \%$ del total de empresarios entrevistados afimaron disponer de información tecnológica, manifestaron recibirla en un $34 \%$ de folletos, $32 \%$ de libros y 
el resto de perídicos y/o revistas, telecomunicaciones y otros medios. Estos resultados llevan a pensar que los empresarios, de alguna manera están informados sobre las nuevas tendencias que en materia tecnológica se llevan a cabo a nivel mundial. Este aspecto es sumamente importante, porque en la medida que conozcan lo que en materia tecnológica se está desarrollando internacionalmente, podrán adoptar esos avances para mejorar sus procesos productivos a fin de poder competir en precio y calidad. De alli que los canales permanentes de información tecnológica, como de mercados y financieros son necesarios para que estas industrias puedan competir en condiciones de igualdad con las industrias internacionales.

En las encuestas realizadas se obtuvo que el $93 \%$ de las pequeñas y medianas industrias metalmecánicas hablan recibido asistencia técnica en los dos últimos años; el resto indicó no haber recibido asistencia técnica. (Gráfico No. 3). Las áreas de mayor apoyo en asistencia técnica fueron las de asesoramiento organizacional con un $40 \%$, seguido de las técnicas de producción e ingeniería con un $32 \%$, un $12 \%$ en mercadeo y finanzas, y el $16 \%$ de industrias respondie-

\section{Gráfico No. 3}

Municipios Maracaibo y San Francisco

Pequeña y Mediana Industria Metalmecánica

Áreas donde han Recibido Asistencia Técnica

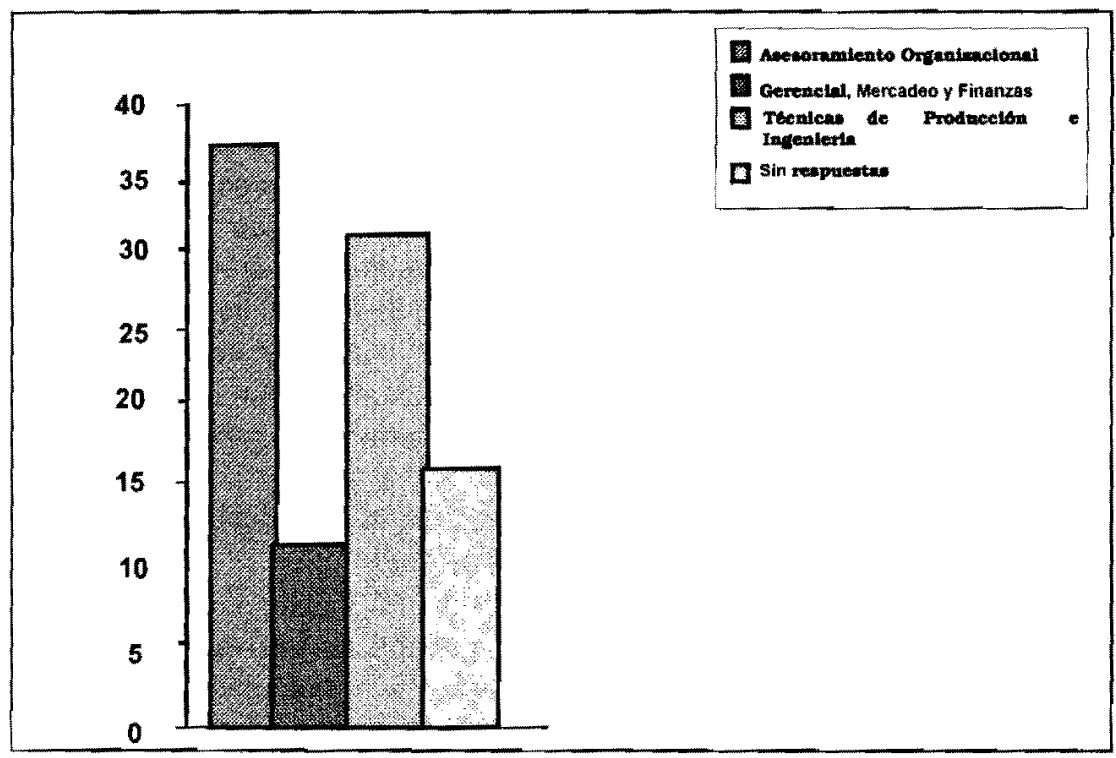

Fuente: Encuesta realizada en los Municipios Maracaibo y San Francisco, Mayo 1997.

Cálculos Propios 
ron no haber recibido ningún tipo de asistencia técnica.

Con referencia al desarrollo tecnológico, la pequeña y mediana industria metalmecánica requiere asistencia en la formación de los recursos humanos, la difusión de información y orientación tecnológica e incentivos de tecnología básica.

Por otra parte, se señalan las instituciones que prestan servicios a la PyMIMM. Las empresas privadas nacionales encabezan con un $43 \%$, seguidas por empresas extranjeras con un $29 \%$, las empresas mixtas con un $17 \%$ y el sector público con un $11 \%$, este último sector es más utilizado por las medianas inferio- res y las pequeñas. Al igual las empresas nacionales siven preferentemente a las pequeñas y mediana superiores.

Aqui se aprecia que el $93 \%$ de los pequeños y medianos industriales metalmecánicos entrevistados contemplan planes de modificación de sus procesos tecnológicos y organizativos. Esto se entiende por los cambios que se están operando en el país en materia económica, lo cual requerirá que las industrias se preparen para ello.

En el gráfico No. 4 se puede observar que los resultados de los proyectos de modificación, presentan dos áreas prioritarias. La optimización de procesos

Gráfico No. 4

Municipios Maracaibo y San Francisco

Pequeña y Mediana Industria Metalmecanica

Finalidad de los Proyectos de Modificación en los Procesos

Tecnológicos y Organizativos



Fuente: Encuesta realizada en los Municipios Maracaibo y San Francisco, Mayo 1997. Cálculos Propios 
o uso de equipos al igual que introducir modificaciones organizativas importantes, ocupando un $24 \%$ cada una, siguiendo con la mejora en diseño de productos con un $20 \%$, un $12 \%$ la disminución de la contaminación, y la sustitución de tecnología de proceso y de materias primas, alcanzan un $10 \%$ cada una.

Actualmente, la PyMIMM, representa un factor de apoyo a la industria petrolera y además se constituye en un centro de innovación tecnológica, puesto que en su tarea por alcanzar mayor productividad, con la mejor calidad y el menor costo posible, introduce nuevos procedimientos y procesos, basados específicamente en la implantación de normas internacionales de calidad ISO (Internatio- nal Standard Organization), exigidas por la industria petrolera ya que su validez es a nivel internacional, por ser las normas que aseguran la calidad de gestión de una empresa sin importar a cual área productiva se dedique, garantizando la excelencia y confianza, esto a su vez disminuye las barreras que impone el comercio internacional, permitiéndoles alcanzar niveles de calidad para competir con el producto importado que en la actualidad esta ingresando al país, a través, del proceso de apertura petrolera.

Unido al anterior señalamiento, las PyMIMM objeto de análisis, realizan revisión de su capacidad de producción, enfatizando en las políticas de seguridad industrial, seguido de estudios relaciona-

Gráfico No. 5

Municipios Maracaibo y San Francisco

Pequeña y Mediana Industria Metalmecánica

Estudios de Capacidad de Producción Actualizados

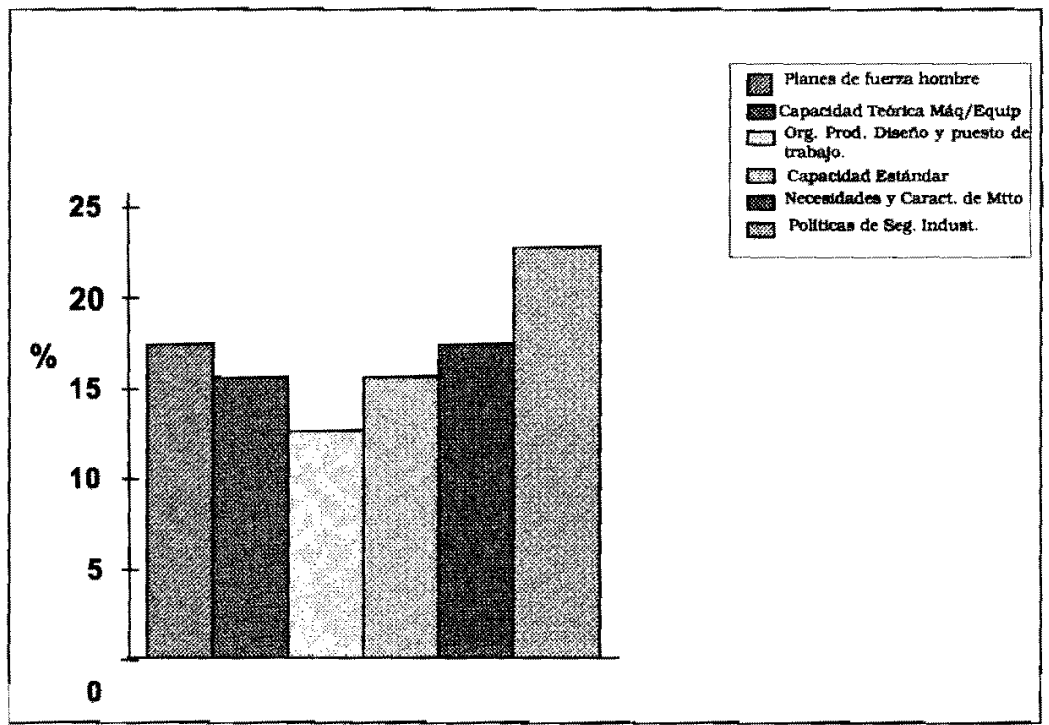

Fuente: Encuesta realizada en los Municipios Maracaibo y San Francisco, Mayo 1997. Cálculos Proplos. 
dos con la capacidad técnica de la maquinaria y equipos, organización de la producción, necesidades y caracterización del mantenimiento y planes de fuerza hombre y puestos de trabajo. Logra así crear un ambiente confiable y mantener un sistema de calidad que fortalezca su propia competitividad, permitiéndoles conseguir la calidad deseada del producto en forma rentable. (Gráfico No. 5)

La mejora de la competitividad estaría basada en capacitar al personal y contratar mano de obra calificada, cambios de maquinarias y equipos, mejoras on la calidad del producto, ampliación de la industria y mejoras organizativas.

Por otra parte, de acuerdo a la respuesta dada por los empresarios, las in- versiones que realizarían en los próximos años, serían: en primer lugar las inversiones intangibles ubicándose en un $58 \%$, seguidas de las inversiones tangibles con un $22 \%$ y por último las innovaciones tecnológicas con un $21 \%$. (Gráfico No. 6)

Dentro de los 14 factores externos al establecimiento que a juicio de los empresarios constituyen un freno al desarrollo de los mismos, están caracterizados como "deficientes servicios técnicos industriales", con un $4 \%$ de participación del total; éste es superado ampliamente por la "insuficiente demanda en su producto" con un $9 \%$, "problemas financieros" con un $12 \%$, "competencia desleal de otras empresas" con un $12 \%$, "problemas con las materias primas" con un

Gráfico No. 6

Municipios Maracaibo y San Francisco

Pequeña y Mediana Industria Metalmecánica

Inversiones de la Industria en los próximos años

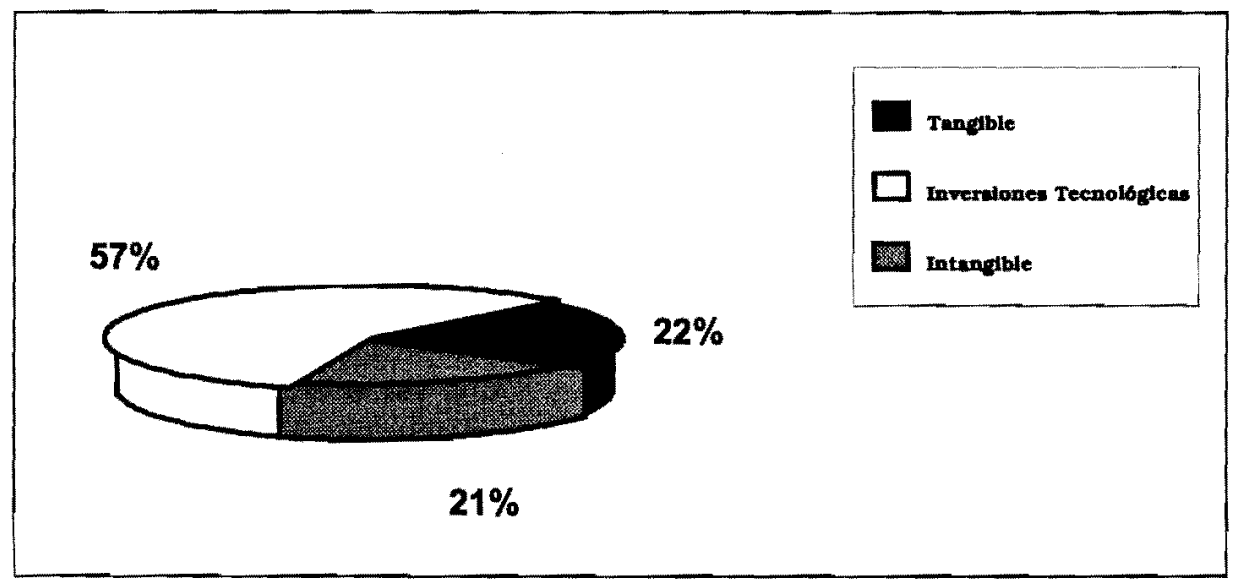

Fuente: Encuesta realizada en los Municipios Maracaibo y San Francisco, Mayo 1997.Cálculos Propios. 


\section{Gráfico No. 7}

Municipios Maracaibo y San Francisco

Pequeña y Mediana Industria Metalmecánica

Factores Externos que han Constituido un freno al

Desarrollo de la Industria

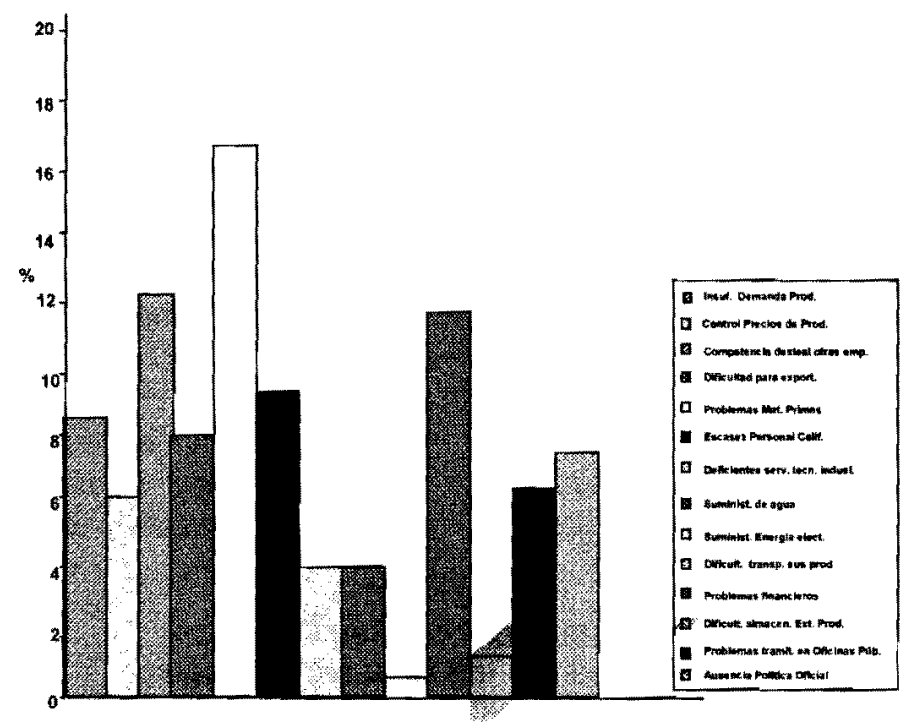

Fuente: Encuesta realizada en los Municipios Maracaibo y San Francisco, Mayo 1997.

Cálculos Propios

17\%. (Gráfico No. 7). Estas respuestas pueden llegar a interpretarse de diferentes maneras: que existen muchos problemas más resaltantes, que los vinculados a la asistencia técnica, que los servicios técnicos no constituyen una prioridad para el funcionamiento de los establecimientos o que los servicios existentes son eficientes.

En el gráfico No. 8 se muestran los principales factores internos que afectan a la PyMIMM, como son la insuficiencia de equipos y maquinarias, dificultad para lograr un eficiente mantenimiento, problemas de adaptación de equipos y maquinarias, problemas de administración de costo y problemas laborales. $\operatorname{Sin}$ embargo, es de destacar que de las 28 industrias entrevistadas el $21 \%$ manifestó que ninguno de ellos constituye un factor interno que frene su desarrollo.

\section{Conclusiones}

Una vez analizada la pequeña y mediana industria metalmecánica ante el proceso de apertura petrolera, es posible exponer las siguientes conclusiones:

1. De acuerdo a la opinión de los industriales entrevistados las características que tiene el proceso de apertura petrolera son las siguientes: 
Gráfico No. 8

Municipios Maracaibo y San Francisco

Pequeña y Mediana Industria Metalmecanica

Factores Internos que han Constituido un freno al

Desarrollo de la Industria

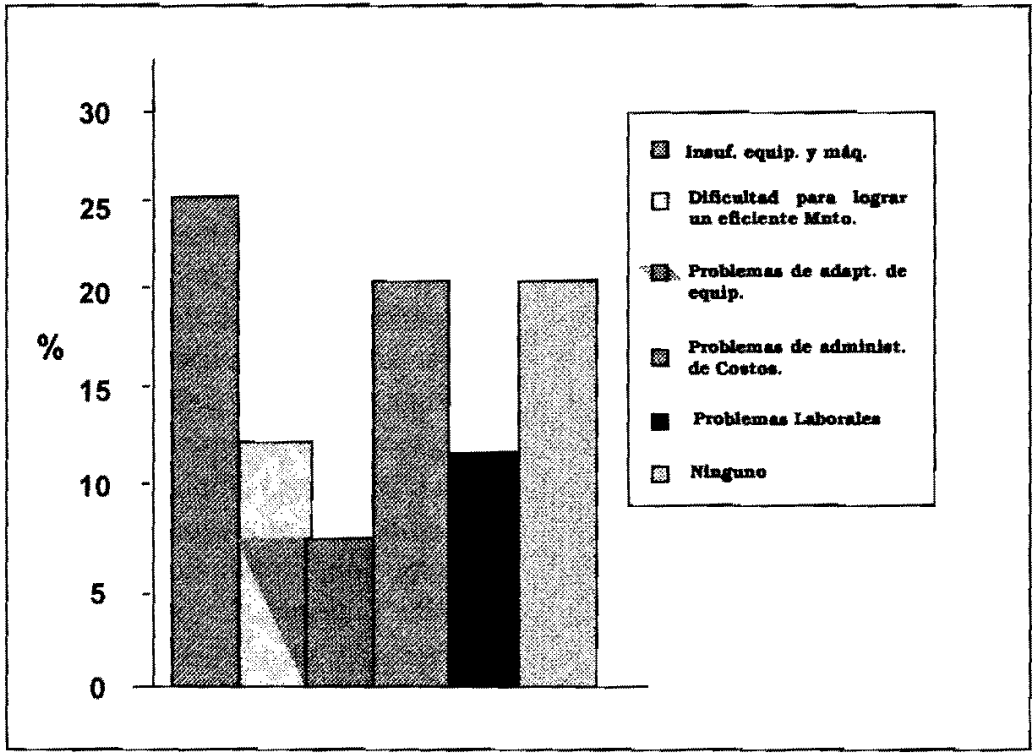

Fuente: Encuesta realizada en los Municipios Maracaibo y San Francisco, Mayo 1997. Cálculos Propios

- Proporciona mejoras socioeconómicas, ya que se contará con inversiones nacionales y foráneas.

- Fomenta la competitividad y reactiva la economía estancada

- Provoca nuevas inversiones con capitales nuevos y dinamiza la economía.

Gran oportunidad de apertura económica que puede ser aprovechada si se crea una conciencia nacional.

- Es uno de los factores que repercutirá en el alto grado de competitividad que tendrán las industrias metaimecánicas y como resultado de esto una mayor calidad del producto y así cumplir con las exigencias petroleras.

- Es fructífero para el pais, ya que existe gran oportunidad de empleo, mayor crecimiento y riqueza.

- Es una gran oportunidad para el desarrollo nacional, ya que generará empleo y reactivará una parte de la industria, específicamente la metalmecánica.

2. Dentro de la pequeña y mediana industria metalmecánica se deben operar cambios significativos para poder dar respuesta a la demanda surgida con el desarrollo petrolero. Es necesario tener presente que la nueva dimensión del nego- 
Industria metalmecánica y apertura petrolera

Caterina Clemenza y col.

cio petrolero crea necesidades de formación gerencial, cada vez con un mayor nivel de excelencia. Se debe lograr que el recurso humano esté capacitado y formado para llevar a la empresa hacia un futuro exitoso.

3. La mayor ocupación del sector, está concentrada en la mediana industria. Probablemente por ser ésta quien brinda mayor oportunidad laboral, ya que cuenta con un parque tecnológico capaz de incorporar un mayor número de trabajadores, cabe destacar que los emplea. dos de la PyMIMM objeto de estudio, se encuentran ubicados en los niveles técnicos y universitarios, lo cual indica que las personas empleadas en este sector se están capacitando para poder enfrentar los retos que implica la nueva realidad económica del país.

4. Según los entrevistados la gestión tecnológica se puede aplicar más efectivamente para mejorar los procesos de producción y modernización eficaz del equipo. Otras áreas en que los empresarios suponen que la gestión tecnológica puede apoyar a sus empresas, incluyen la capacitación de los empleados el aumento de las ventas y el perfeccionamiento de la calidad de sus productos y servicios.

5. Las pequeñas y medianas industrias metalmecánicas (PyMIMM) que deben enfrentarse a las exigencias de clientes como las industrias públicas y privadas, requieren que sus proveedores dispongan de tecnología actualizada, que cumplan con las normas internacionales de producción y que ofrezcan productos de calidad a precios competitivos. En este aspecto se plantea que la PyMIMM deben diseñar la estrategia tecnológica que se adecúe a sus intereses y de esta manera lograr ser más competitivos.

6. Al respecto se puede indicar que la estrategia tecnológica podría definirse alternativamente como el patrón fundamental de acciones de una organización orientada a uso de la tecnología disponible, bien sea desarrollada internamente (a través de actividades de investigación y desarrollo) o foránea, obtenida a través de licencias o adquisiciones, la finalidad es satisfacer las necesidades actuales y potenciales de los clientes, obtener una ventaja sostenible sobre los competidores, facilitar e interpretar las oportunida. des e impactos competitivos en términos de productos, procesos, materias primas, distribución, información, servicio al cliente, proveedores, organización, administración y mercadeo.

7. Se requiere también una estrategia competitiva que según Porter es el conjunto de acciones defensivas y ofensivas que realiza la empresa para crear una posición defendible en un sector industrial, enfrentar con exito las fuerzas competitivas y maximizar el valor de las capacidades que la distinguen de sus competidores. La competitividad es un concepto fundamental en las empresas que persiguen como objetivo central mantener y aumentar su participación en el mercado. Para hacerlo es necesario prepararse puesto que la competitividad no es un don natural, es una capacidad que solamente se adquiere con esfuerzo y dedicación. Si la empresa no es competitiva no puede sobrevivir, menos aún progresar en un ambiente signado por cambios profundos como es el ambiente de los negocios.

8. Las PyMIMM deben revisar la manera como hacen sus operaciones y 
es así necesario, cambiar los métodos que utilizan para manejar el negocio y adaptarse a las nuevas exigencias del mercado nacional, aunado a la aplicación de las normativas de calidad y productividad (Normas ISO - 9000) para mejorar la calidad de gestión a nivel gerencial y a nivel productivo, partiendo del mejoramiento del recurso humano y de los procesos productivos. En tal sentido, deben realizar esfuerzos y destinar recursos para mejorar la capacidad de producción, tecnología de productos y procesos a traves de la certificación ISO - 9000, que es sumamente importante para lograr los estándares de calidad exigidos por la industria petrolera a las empresas privadas y extranjeras a fin de brindarle confianza a sus cliente en cuanto a la calidad de sus productos y senvicios.

\section{Referencias bibliográfícas}

Bitar, Sergio y Troncoso Eduardo. (1993). "El Desafio Industrial de Venezuela". Edt. Pomaire Argentina, pág 168.

Banco Central de Venezuela (1995). Boletín Económico.

CEPAL (1989). "La Industria de Bienes de Capital en América Latina: Realidad y Potencialidades. Junio, pág 5.
FERRER, Jullana (1990). Diagnóstico de la Competencia Industrial. Facultad de Clencias Económicas y Sociales. División de Estudios para Graduados. Tesis. pág 192.

González y Jiménez. (1990) "Las pequeñas y medianas empresas y algunas consideraciones prácticas". Revista Venezolana de Gestión. Investigación y Gerencia. N225. Vol. VI. No3. Pág. 138-145.

Katz, Jorge, (1983). “El Escenario Tecnológico Latinoamericano en el Campo Metalmecánico". Revista CEPAL, Abril, pág. 101.

Marval, Oscar. (1994). "Importancia y Desarrollo de la Pequeña y Mediana industria (PyMI)". CENDES, Universidad Central de Venezuela. Pág. 8-9.

Paredes, Lombardo, (1997). "Los proyectos de PDVSA generarán un promedlo de 45.000 empleos directos". Diario El Nacional. Caracas. Pág. E1.

Peñaloza, Humberto (1996). "Apertura Petrolera y Desarrollo Nacional". Revista Barriles año 7 Marzo - Abril. № 43 , pág. 36.

Petróleos de Venezuela (PDVSA). (1997). "Informe Anual)

Nueva Economia. (1997). Academia Nacional de Ciencias Económicas. Año 6 No. 8, Abril, Caracas - Venezuela. 